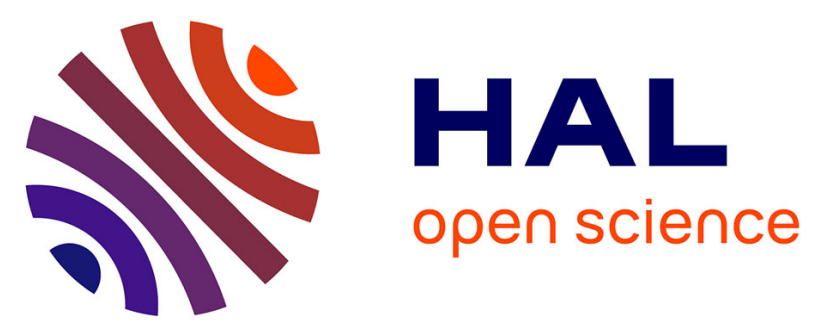

\title{
Defect analysis and excitons diffusion in undoped homoepitaxial diamond films after polishing and oxygen plasma etching
}

Pierre-Nicolas Volpe, Pierre Muret, Franck Omnès, Jocelyn Achard, François Silva, Ovidiu Brinza, Alix Gicquel

\section{To cite this version:}

Pierre-Nicolas Volpe, Pierre Muret, Franck Omnès, Jocelyn Achard, François Silva, et al.. Defect analysis and excitons diffusion in undoped homoepitaxial diamond films after polishing and oxygen plasma etching. Diamond and Related Materials, 2009, 18 (10), pp.1205-1210. 10.1016/j.diamond.2009.04.008 . hal-00967297

\section{HAL Id: hal-00967297 https://hal.science/hal-00967297}

Submitted on 31 Mar 2014

HAL is a multi-disciplinary open access archive for the deposit and dissemination of scientific research documents, whether they are published or not. The documents may come from teaching and research institutions in France or abroad, or from public or private research centers.
L'archive ouverte pluridisciplinaire HAL, est destinée au dépôt et à la diffusion de documents scientifiques de niveau recherche, publiés ou non, émanant des établissements d'enseignement et de recherche français ou étrangers, des laboratoires publics ou privés. 


\title{
Defect analysis and excitons diffusion in undoped homoepitaxial diamond films after polishing and oxygen plasma etching
}

\author{
Pierre-Nicolas Volpe ${ }^{* 1}$, Pierre Muret ${ }^{1}$, Franck Omnes ${ }^{1}$, \\ Jocelyn Achard², François Silva ${ }^{2}$, Ovidiu Brinza ${ }^{2}$, Alix Gicquel ${ }^{2}$ \\ ${ }^{1}$ Institut Néel-CNRS \& Université Joseph Fourier, BP166, 38042-Grenoble-Cedex9, France \\ ${ }^{2}$ LIMHP-CNRS, Université Paris 13, 99 avenue Jean Baptiste Clément, 93430 Villetaneuse, France \\ Received XXX, revised XXX, accepted XXX \\ Published online XXX
}

Keywords : homoepitaxial diamond films, defects, excitons, luminescence, polishing, etching

\begin{abstract}
Several $65 \mu \mathrm{m}$ thick epitaxial diamond films prepared on (100) Ib substrates by high power, pulsed microwave plasma assisted chemical vapour deposition (HP-PMPCVD) are studied as a function of surface treatments by cathodoluminescence (CL) and photoluminescence (PL) spectroscopies. They are either as-grown, or polished, or etched by a microwave oxygen plasma, or after applying subsequently the two last processes. In CL spectra, bands due to defects occur at $2.3 \mathrm{eV}, 3.07 \mathrm{eV}, 3.6 \mathrm{eV}$ and $4.7 \mathrm{eV}$, the last one being specific of polished surfaces, with nearly no contrast in the luminescence image, contrary to other CL images. A fundamental result consists in demonstrating that the defects induced by polishing can be removed by oxygen plasma etching. Additionally, in order to assess how luminescence spectra originate from a peculiar depth or not, a bevelled sample is studied. PL spectra are acquired on the sample side while CL spectra are measured at several points on the bevel top side till to the Ib substrate. Comparison of the two sets of result show that the $\mathrm{H} 3$ signal originates from the Ib substrate even if it is present in the CL spectra of the film. An analysis of the change in the intensity of the TO free exciton line, defect bands and H3 signal, along decreasing photon energies, as a function of the thickness of the remaining HP-PMPCVD film, is performed with the help of a model taking the diffusion of the unrecombined excitonic pairs and the re-excited photoluminescence into account. CL images recorded at specific wavelengths, which do not show inverted contrast, are also assessed. From these data, the exciton diffusion length is evaluated to $11 \mu \mathrm{m}$ in the major part of the epitaxial layer except for the first $20 \mu \mathrm{m}$ close to the Ib substrate where it decreases down to $2 \mu \mathrm{m}$. This study sheds light on the interpretation of luminescence spectra excited by an electron beam in undoped diamond layers. Defects bands due to damages induced by polishing and etching processes are also documented.
\end{abstract}

\section{Introduction}

Diamond, thanks to its overwhelming electrical, mechanical and chemical properties, offers important perspectives for electrical [1-4] and optical [5-7] applications. Meanwhile, some features such as the non uniform film thickness or surface roughness obtained after crystal growth by chemical vapour deposition (CVD) are limiting factors for these applications. Then, the diamond substrates and films have to be polished. For several years, a huge number of polishing techniques applied to diamond has been developed: mechanical lapping [1], chemically assisted mechanical polishing [2], thermo-chemical polishing [3], laser and ion beam polishing [4,5]. The use of the chemically assisted mechanical polishing process, also known as "scaife polishing", creates on the diamond surfaces a lot of oriented striations 10 to 15 nanometres deep, which can be clearly detected by Nomarski analysis or AFM measurements. The surface of the diamond single crystal seems to be very flat and has a uniform roughness over all the (100) growth sectors. Meanwhile, it

"Corresponding author; e-mail: pierre-nicolas.volpe@grenoble.cnrs.fr, Phone: +33 (0)4 76887469 
has been showed in the several previous studies that the polishing defects of the diamond substrate propagate into homoepitaxial layers, leading to plastic deformations and trapping of carriers into deep defects [8-13]. In a previous study [14], it has been showed that a plasma etching treatment applied to the substrate before film growth can remove defects of the $\mathrm{Ib}(100) \mathrm{HPHT}$ diamond substrates and induces an increase of the homoepitaxial diamond film quality.

The present study aims at identifying, by cathodoluminescence (CL) analysis, the defects induced either by the "scaife" polishing or ECR etching on a (100) oriented, $65 \mu \mathrm{m}$ thick, intrinsic CVD diamond films. The purpose is also to check the optimised etching conditions previously published [15]. Moreover, CL and photoluminescence (PL) spectroscopies are performed on a bevelled sample in order to assess where the luminescence signals come from. We show here that, in case of intrinsic CVD layers, the excitons diffuse out of the primary electrons interaction volume (PEIV), as it has already been mentioned in diamond layers $[16,17]$. This effect induces an increase of the depth probed by CL far beyond the PEIV. The excitonic diffusion length can be determined, thanks to a simulation model based on unrecombined excitonic pairs diffusion and re-excited photoluminescence. For an acceleration voltage of $10 \mathrm{kV}$, while the PEIV extends about $1 \mu \mathrm{m}$ below the surface, the depth probed by CL has been evaluated to 11 microns at the top of the $65 \mu \mathrm{m}$ thick layer, whereas it reduces to $2 \mu \mathrm{m}$ in the first $20 \mu \mathrm{m}$ close to the Ib substrate. This result shows that a more defective layer is grown at the beginning of the growth process, in accordance with Tallaire et al. results [18].

\section{Experimental}

The CL analysis have been performed on $65 \mu \mathrm{m}$ thick undoped diamond layers deposited by HP-PMPCVD on Ib (100) diamond substrates $\left(3 \times 3 \times 0.5 \mathrm{~mm}^{3}\right)$ provided by Sumitomo company. Some of these "as-grown" samples have been either polished by "scaife" polishing or etched by pure oxygen ECR plasma under optimised conditions [15] during 1 hour, an operation which removed from 3 to $4 \mu \mathrm{m}$ of the diamond layer. The detailed description of the ECR equipment has been already done elsewhere [19, 20]. After growth, the surface of one of the samples has been bevelled by mechanical polishing with an angle of 5 degrees. The CL analysis have been performed at $5 \mathrm{~K}$ with a Quanta 200 secondary electron microscope (SEM), coupled with a collecting mirror and a CCD array through a HR 460 JOBIN YVON spectrometer. Concerning the bevelled and polished sample, the CL analysis have been either made with an acceleration voltage in the range $5-30 \mathrm{kV}$ in order to change the depth probed by primary electrons, according to the Davies law [21], or with constant voltage of $10 \mathrm{kV}$, constant spot size and exposure time, along the $0_{\mathrm{x}}$ axis (see Fig.1), every $100 \mu \mathrm{m}$ from the edge between the unpolished and flat part of the CVD layer and the bevelled part.

Photoluminescence has been performed in a micro-Raman JOBIN YVON HORIBA LabRam HR800 confocal spectrometer, using a $\times 100$ objective, which yields a spot of about $1 \mu \mathrm{m}$ diameter, and the $488 \mathrm{~nm} \mathrm{Ar}+$ laser excitation.

\section{Cathodoluminescence analysis of as-grown, polished and oxygen plasma etched homoepitaxial diamond thick films}

In order to evidence the effect of polishing, CL spectra are taken first on an as-grown film and secondly on the same film after a "scaife" polishing operation, which aims at giving a mirror surface like suitable for jewellery, without any special care for minimizing other detrimental effects. In this section, CL spectra are all normalized on the maximum intensity of the $\mathrm{FE}_{\mathrm{TO}}$ line. On figure 2-(a), it can be seen that the polishing process made on an as-grown CVD layer (S63) enhances the amplitude of the defect bands by a factor of 3 to 4 decades. These bands are located at 2.32 (green A band), 3.05 (A band), 3.7 and $4.7 \mathrm{eV}$. The last band was not present before polishing, which implies that it has been created by the polishing treatment, and should then attributed to the presence of highly defective crystalline defects in the sub-surface region. The Fig 2-(b) shows that the polishing treatment also increases the full width at half maximum (FWHM) of the excitonic peaks by a factor 3 to 4 and that the $\mathrm{FE}_{\mathrm{TA}}$ and $\mathrm{FE}_{\mathrm{LO}}$ lines cannot be detected anymore. From these results, it is now obvious that the polishing treatment is a very destructive process and induces a very damaged zone in the sub-surface of the sample. The thickness of this damaged layer can be roughly estimated to some micrometers from the depth probed by CL as discussed in the next section. 
Figure 3 shows CL spectra taken on the same sample, firstly on the as-grown surface, then after polishing and finally after the removal of $4.25 \mu \mathrm{m}$ of the polished sample by oxygen plasma etching. It can be clearly seen on Fig 3-(a), that a sequential "polishing and etching" treatment, under optimised conditions [15], permits to cancel all the defect bands amplified or created by the polishing process and to restore or even to improve the initial spectrum. The enhancement of the crystalline quality can be better seen on the excitonic part of the spectrum in (III) of Fig. 3-(b), where the peak FWHMs are reduced by a factor 3 to 4 , down to $8 \mathrm{meV}$, much narrower than for the initial surface. Consequently, it is possible to get comparable or even higher crystalline quality than before polishing for the first few micrometers of the diamond layer. However, the comparable intensity of the $\mathrm{FE}_{\mathrm{LO}}$ and $\mathrm{FE}_{\mathrm{TO}}$ excitonic lines suggests that some imperfections have been created by the etching process, because in ideal diamond crystals, the $\mathrm{FE}_{\mathrm{LO}}$ is usually less intense. In comparison to curves (I) and (II) of Fig 3-(b), the increase of the FE $_{\mathrm{LO}}$ line intensity in curve (III) could be induced by a change in the exciton-lattice interaction potential at some new defect centers, due to the ion bombardment during the etching process.

CL images have been recorded at several wavelengths in order to detect the homogeneity of the various lines and defect bands and are displayed on Fig.4 (the lighter the area, the higher the luminescence intensity) together with the topographic image delivered by secondary electrons. Most of the defects cannot be seen in the topographic image but appear in CL images at several wavelengths except for the image taken at $4.7 \mathrm{eV}$, which is nearly uniform. This last fact correlates well with a polishing induced origin for this band, since the defects responsible for its presence in the CL spectra are located within a few micrometers under the surface and are not related to the defects induced by the substrate. The H3 signal (Fig. 4) comes from the nitrogen buried into the substrate, because signals which may originate from nitrogen in the CVD layer are emitted at other energies $(2.16 \mathrm{eV})$. Then, the correlated features seen on the other images taken respectively at $2.98 \mathrm{eV}, 3.7 \mathrm{eV}$ and $5.27 \mathrm{eV}$ are induced by the irregularities linked to the $\mathrm{H} 3$ contrast. All these four signals are weakened along this straight line. For the signal coming near $2.467 \mathrm{eV}$ however, the green $\mathrm{A}$ and A bands, which are nearby in the spectrum of the polished sample (Fig. 2-(b) I), probably contribute to the image. Therefore, one can wonder whether this straight line may be either reminiscent of a linear defect of the substrate, like striations previously quoted or due to the final polishing process. But in this second case, the same contrast would also appear in the image of the $4.7 \mathrm{eV}$ band, which is not true. Hence, the most probable interpretation of these images is that the $4.7 \mathrm{eV}$ band comes from the last polishing process while other bands or lines come from defects induced by the Ib substrate. Such a fact suggests first that defects originating from the substrate can propagate through the whole layer and secondly that all the luminescence signals may not come only from the PEIV. On the contrary, if all the light comes from the PEIV, the balance of the various recombination intensities would favour some of them at the expense of others, and would produce inverted contrast for some images in comparison to others, like seen in CVD boron doped layers $[9,15]$ with thickness lower than ten micrometers. The case of the H3 lines is specially important, since nitrogen is not expected in the high purity CVD layer, whereas it exists at concentration up to a few hundreds ppm in the Ib substrate. Therefore, a deeper analysis is needed first for checking the last assumption and secondly for a detailed understanding of the physical phenomena involved in the recombination of excitons and other luminescence effects.

\section{Analysis of the light emission location in a bev- elled layer from $P L$ and $C L$ spectroscopies}

In order to confirm that the $\mathrm{H} 3$ lines of the CL spectra do not originate from the CVD layer itself, but only from the Ib substrate, photoluminescence (PL) spectroscopy with a sub-band gap excitation energy $(2.54 \mathrm{eV})$, is performed along the side of the sample, at a location where the whole thickness $(65 \mu \mathrm{m})$ of the CVD layer still exists. Such an excitation is obviously unable to create excitons. Figure 5 clearly shows that a PL peak appears at $503 \mathrm{~nm}$ like in CL spectra for the main H3 line, while in (a) the interface and in (b) the Ib substrate are probed, but completely vanishes as soon as the CVD layer is probed, only $1 \mu \mathrm{m}$ above the interface, like in (c), or above in (d). Then, in addition to the suggestions coming from CL images, the absence of the H3 line in the PL spectrum of the CVD layer needs to consider the propagation of both excitons and light outside the PEIV for interpreting the CL spectra and specially the presence of the H3 line in them. According to the energy of the emitted photons and the location of the luminescent transitions, several mechanisms have to be taken into account : (i) the excitonic recombination inside the PEIV, which is about $1 \mu \mathrm{m}$ deep at the $10 \mathrm{kV}$ acceleration voltage ; (ii) the excitonic recombination outside the PEIV, due to diffusion of previously unrecombined excitonic pairs; (iii) the photoluminescence due to the possible excitation of valence band electrons to 
deep levels in the band gap by the UV light generated by the excitons or other recombinations, followed by radiative transitions to valence band states. Mechanisms (i) and (ii) would give all the radiative transitions while the mechanism (iii) would involve only photons with energies lower than the excitonic lines. No normalization of the CL intensities is done, except for the measurement duration. Spectra are measured for thickness $t$ from 0 to $65 \mu \mathrm{m}$ and shown in Fig. 6 for thickness $t$ from 11 to $65 \mu \mathrm{m}$. It must be noticed that defect bands are much less prominent in this bevelled sample than in samples studied in section 3, although a similar polishing method, but manual, has been used. As mentioned in ref. [22], polishing may have very different effects according to the polishing set-up and operator skill .

All the excitonic pairs which reach the substrate are supposed to give only recombinations other than exciton lines since the intensity of these exciton lines becomes negligible when the Ib substrate is directly hit by the electron beam. Moreover, the unrecombined excitonic pairs are assumed to diffuse uniformly in the whole $2 \pi$ steradians solid angle from the impact point of the primary electrons, and their concentration is supposed to follow an exponential decay law along the radius $r$, with a diffusion length $\lambda$. Then, the $\mathrm{FE}_{\mathrm{TO}}$ intensity $I_{\text {FETO }}(t)$ written in equation (1) results from the sum of a constant term $I_{P E I V}$ due to light emitted inside the PEIV, and a term which depends on the thickness $t$ of the CVD layer. This second term is the $\mathrm{FE}_{\mathrm{TO}}$ intensity outside the PEIV, calculated with the previous hypothesis by integrating the exciton concentration in all the directions making an angle $\theta$ with the normal to the surface, along a path going from a radius $r=0$ to $r=t / \cos \theta$ until the flat interface between the CVD layer and the Ib substrate is reached, if the dimensions of the PEIV are neglected.

$$
I_{\text {FETO }}(t)=I_{\text {PEIV }}+I_{0} \int_{0}^{\pi / 2} \int_{0}^{t / \cos \theta} \exp (-r / \lambda) \times 2 \pi r^{2} d r d \theta
$$

The exciton diffusion length $\lambda$ is the main adjustable parameter in equation (1) because it strongly influences the shape of the fitting curve, as shown in Fig. 7-(a). As it can be seen, the four first points can be fitted with $\lambda=2 \mu \mathrm{m}$ up to a thickness of $20 \mu \mathrm{m}$ while $\lambda=11 \mu \mathrm{m}$ is well appropriate for thickness between 20 and $65 \mu \mathrm{m}$. The last two points suffer more dispersion probably because measurements are done very close to the edge of the bevel where defects may be different from those encountered along the bevel itself. This change in the excitons diffusion length or mean free path may be either due to a better quality of the CVD layer for thickness larger than $20 \mu \mathrm{m}$ or to a higher boron content nearer the $\mathrm{Ib}$ substrate as in ref. [18]. Indeed, boron concentrations near or in excess of a few $10^{16} \mathrm{~cm}^{-3}$ are expected to strongly decrease the diffusion length of the free excitons since excitons bound on neutral boron atoms are more and more probable, with interatomic distances between boron atoms much less than $1 \mu \mathrm{m}$. The simulation turns out not to be sufficiently accurate for assessing the first term $I_{P E I V}$, which will be considered as negligible in equation (1).

The intensities of the A band and of the set of the H3 lines, the main ones after the exciton lines in the spectra of Fig. 6 , excepted for $t=65 \mu \mathrm{m}$, are respectively fitted with equations (2) and (3), without any change of the values previously found for the exciton diffusion lengths $\square \lambda$. This is done only to check the possibility of finding actually constant parameters $I_{A 0}, \alpha \square, \quad \beta, \gamma, \delta$ and $\square \varepsilon$ which comply with the experimental data (Fig. 7-(b) and 7-(c)).

$$
\begin{gathered}
I_{A}(t)=I_{A 0}+\alpha\left[I_{\text {FETO }}(\infty)-I_{\text {FETO }}(t)\right]+\beta I_{\text {FETO }}(t) \\
I_{H 3}(t)=\gamma\left[I_{F E T O}(\infty)-I_{F E T O}(t)\right]+\delta I_{\text {FETO }}(t)+\varepsilon I_{A}(t)
\end{gathered}
$$

The A band intensity is supposed to be due to three terms: $I_{A 0}$ is the A band intensity emitted inside the PEIV and assumed to be constant at the surface of the bevel; the second term in equation (2) is proportional through the $\alpha$ constant factor to the excitonic pairs which have not experienced a radiative transition at $5.26 \mathrm{eV}$ and may recombine through transitions at lower energies; the third term is due to the photoluminescence excited by UV light travelling through the whole CVD layer and coming from excitonic lines. The integrated H3 lines intensity is supposed to originate only from the $\mathrm{Ib}$ substrate and is given by equation (3) with three terms: the first one has the same origin as the second one in equation (2); the two last are due to photoluminescence excited respectively by the UV light coming form excitonic lines and the A band inside the CVD layer. It is necessary to stress that the diffusion length is the only fitting parameter of data which controls the shape of the simulated curve in Fig. 7-(a). Then, it is only applied (with other fitting parameters contained in equations 2 and 3) to the other sets of data in Fig. 7- (b) and (c). In this Fig. 7-(a), it is clearly showed that fitting the data in the region of $20 \mathrm{um}$ from the film/substrate boundary with an excitonic diffusion length of 11 microns is not possible and that the curve simulated with an excitonic diffusion length of $2 \mu \mathrm{m}$ is necessary for matching the four first points. 
From the simulation results, one can deduce the relative strength of the various luminescence intensities in equation (2). For the $\lambda=2 \mu \mathrm{m}$ case, valid for thickness below $20 \mu \mathrm{m}$, the first and second terms are negligible, indicating that photoluminescence is still dominant. For the $\lambda=11 \mu \mathrm{m}$ case, valid for thickness from 20 to $65 \mu \mathrm{m}$, the third term is growing from $50 \%$ to $100 \%$ while the second one is decreasing from $50 \%$ to $0 \%$. The trends are the same for the three terms in equation (3). The first term, related to the cathodoluminescence intensity due to excitons which recombine inside the substrate, amounts to $98 \%$ for thickness below $2 \mu \mathrm{m}$, and decreases finally to zero for the maximum thickness. Conversely, the two last terms, due to photoluminescence, increase with the thickness and their sum becomes dominant for $t>30 \mu \mathrm{m}$.

As a conclusion, this analysis demonstrates that the photoluminescence excited by the light coming from the $\mathrm{FE}_{\mathrm{то}}$ recombination become prominent in the whole emitted spectrum as soon as the thickness of the probed homoepitaxial layer exceeds some mean free paths of excitons. Concurrently, because this condition is achieved for $65 \mu \mathrm{m}$ thick layers, if the $\mathrm{FE}_{\mathrm{TO}}$ recombination is weaken in some area, less light is emitted and hence the other photoluminescence signals are weaker too, explaining the similar contrasts in the spectral images of Fig. 4. It must be stressed that this whole picture is valid for thick undoped layers but would completely change in thin doped layers.

\section{Conclusions}

Cathodoluminescence studies performed on $65 \mu \mathrm{m}$ thick homoepitaxial undoped layers of diamond show that polishing may induce strong damages near the surface, testified by the appearance or increase of defect bands in the spectra and anomalous width of exciton lines. A subsequent dry etching treatment in an ECR oxygen plasma reactor under optimized conditions may restore the defect bands intensity at its lowest level and even improve the exciton lines width. So, it would be useful to remove some hundreds of nanometres of the as-grown films in order to get a better crystalline quality near the surface. However, it is difficult to have an accurate knowledge of the depth where light is emitted in these thick undoped films because photoluminescence often dominates cathodoluminescence intensity, due to the excitons mean free path. In this way, luminescence signals can come from any depth across the whole thickness and even mainly from the substrate, like in the $\mathrm{H} 3$ case. Finally, we have measured the excitons mean free path from the comparison of the $\mathrm{FE}_{\mathrm{TO}}$ line intensity to the data given by a diffusion model. It turns out to be $11 \mu \mathrm{m}$ in the 50 or $60 \mu \mathrm{m}$ of the film close to the surface and $2 \mu \mathrm{m}$ in the first 15 or $20 \mu \mathrm{m}$ close to the substrate.

Acknowledgements: The authors wish to thank J. E. Butler (NRL Washington, USA) who did the bevel on the last sample and polished it, F. Donatini (Néel Institut, CNRS, Grenoble, France) for his assistance in cathodoluminescence measurements, the Rhône Alpes Regional Council (France) and the "Agence Nationale pour la Recherche" (France) through the contract "DIAMOOND" 06-2-134411 for their financial supports.

\section{References}

[1] L.Y.S. Pang, S.M.C. Simon, C. Johnston, P.R. Chalker, R.B. Jackman, Diamond Relat. Mat. 6 (1997) 333.

[2] H. Kiyota, E. Matsushima, K. Sato, H. Okushi, T. Ando, M. Kamo, Y. Sato, M. Iida, Appl. Phys. Lett 67 (1995) 3596.

[3] I. M. Buckley-Golder, P. R. Chalker, C. Johnston, S. Romani, M. Werner, in Advances in New Diamond Sci. and Technol.,

Ed. S. Saito, N. Fujimori, O. Fukunaga, M. Kamo, K. Kobashi and M. Yoshikawa (MYU, Tokyo 1994) pp 669 - 678, Proceedings of the Fourth Internat. Conf. on New Diamond Sci. and Technol., Kobe, Japan, July 1994.

[4] D.R. Kania, M.I. Landstrass, L.S. Pan, Diamond Relat. Mat. 2 (1993) 1012.

[5] C. Vivesang, L. Ferlazzo-Manin, M.F. Ravet, G. Turban, F. Rousseau, A. Gicquel, Diamond Relat. Mat. 5 (1996) 840.

[6] C. Chardonnet, V. Bernard, C. Daussy, E. Anger, A. Giquel, Appl. Optics 35 (1996) 6692.

[7] T. Teraji, S. Yoshizaki, H. Wada, M. Hamada, T. Ito, Diamond Relat. Mat. 13 (2004) 858.

[8] H. Watanabe, D. Takeuchi, S. Yamanaka, H. Okushi, K. Kajimura, T. Sekiguchi, Diamond Relat. Mat. 8 (1999) 1272.

[9] T. Teraji, M. Yamamoto and T. Ito, J. Crystal Growth 285 (2005) 130.

[10] T. Teraji, Physica Status Solidi (a) 203 (2006) 3324.

[11] G. Bogdan, M. Nesládek, J. D'Haen, K. Haenen, M. D'Olieslaeger, Diamond Relat. Mat. 15 (2006) 508.

[12] M. Wade, P. Muret, Diamond Relat. Mat. 15 (2005) 614.

[13] P. Muret, M. Wade, Physica Status Solidi (a) 203 (2006) 3142.

[14] A. Tallaire, J. Achard, F. Silva, R.S. Sussmann, A. Gicquel, E. Rzepka, Physica Status Solidi (a) 201 (2004) 2419.

[15] P.-N. Volpe, P. Muret, F. Omnes, Physica Status Solidi, (a) 205 (2008) 2173.

[16] J. B. Cui, J. Ristein and L. Ley, Diamond Relat. Mat. 9 (2000) 1036-1040. 
[17] J. Ristein, W. Stein, and L. Ley, Phys Rev. Lett. 78 (1997) 1803.

[18] A. Tallaire, M. Kasu, K. Ueda, Diamond Relat. Mat. 17 (2008) 506.

[19] M. Bernard, A. Deneuville, T. Lagarde, E. Treboux, J. Pelletier, P. Muret, N. Casanova, E. Gheeraert, Diamond Relat. Mat. 11 (2002) 828.

[20] M. Bernard, A. Deneuville, L. Ortega, K. Ayadi, P. Muret, Diamond Relat. Mat. 13 (2004) 287.

[21] G. Davies, Chem. Phys. Carbon 13 (1977) 113.

[22] T.E. Derry, L. Smit, J.F. van der Veen, Surf. Sci. 167 (1986) 502, and references in T.E. Derry, Nic van der Berg, N.W. Makau, Diamond Relat. Mat. 17 (2008) 127. 


\section{Figures and captions:}

Figure 1: View of the bevelled sample

Figure 2: CL spectra taken at $10 \mathrm{kV}$ and $5 \mathrm{~K}$ on the same CVD layer S63, (I) before and (II) after polishing treatment, in the whole energy range (a) and in the excitonic energy range (b), all normalized on the $\mathrm{FE}_{\mathrm{To}}$ intensity.

Figure 3: CL spectra taken at $10 \mathrm{kV}$ and $5 \mathrm{~K}$ on the same CVD layer S46, (I) before or (II) after a polishing treatment, and (III) after a polishing and etching sequential treatments, along the whole energy range (a) and in the ex citonic energy range (b). All spectra are normalized on the $\mathrm{FE}_{\mathrm{TO}}$ intensity.

Figure 4: SEM and CL images taken at $10 \mathrm{kV}$ on the same $75 \times 90 \mu \mathrm{m}^{2}$ square area of the polished CVD layer S63 whose spectra are displayed in Fig. 2.

Figure 5: PL spectra taken along the side of the beveled sample sketched in Fig. 1, with a CVD layer $65 \mu \mathrm{m}$ thick, at various distances below the surface ; (a) $65 \mu \mathrm{m}$; (b) $70 \mu \mathrm{m}$; (c) $64 \mu \mathrm{m}$; (d) $60 \mu \mathrm{m}$, under $488 \mathrm{~nm}(2.54 \mathrm{eV}) \mathrm{ex}-$ citation. The narrow line is due to the Raman Stokes inelastic scattering at $1332 \mathrm{~cm}^{-1}(165 \mathrm{meV})$ giving a line at $(2.54-0.165) \mathrm{eV}=2.375 \mathrm{eV}(522 \mathrm{~nm})$ in the PL spectrum.

Figure 6: CL spectra taken on the bevelled sample at $10 \mathrm{kV}$ and $5 \mathrm{~K}$, and measured for various thickness of the CVD layer indicated at the right side. Each are shifted of $0.2 \mathrm{eV}$ along the horizontal axis for clarity.

Figure 7: Luminescence intensities of $\mathrm{FE}_{\mathrm{To}}$ (a, squares), $\mathrm{A}$ band (b, circles) and $\mathrm{H3}$ lines (c, diamonds), as a function of the thickness of the bevelled sample, and comparison with the simulation curves calculated from the diffusion model (lines).

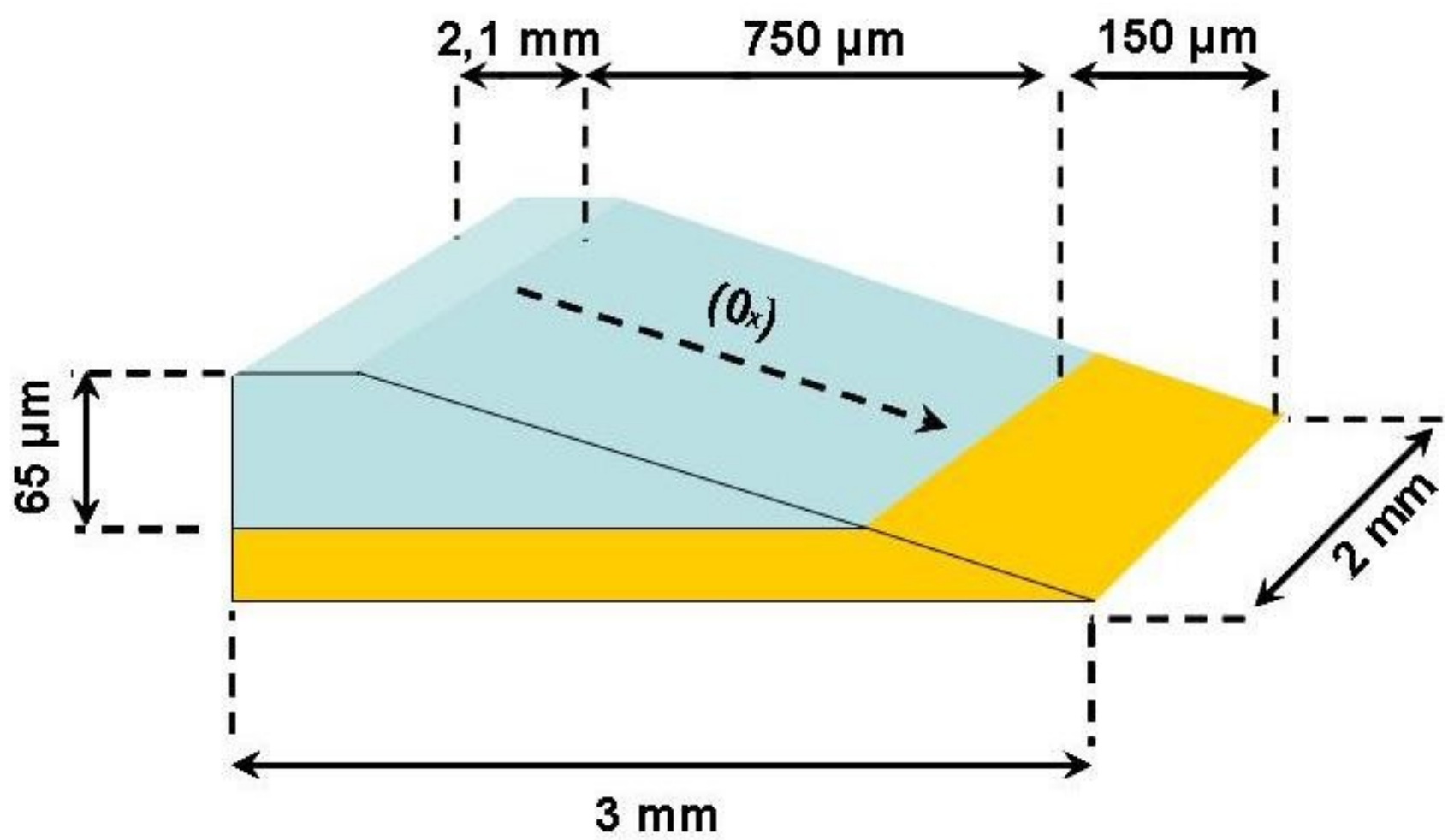

Fig. 1 

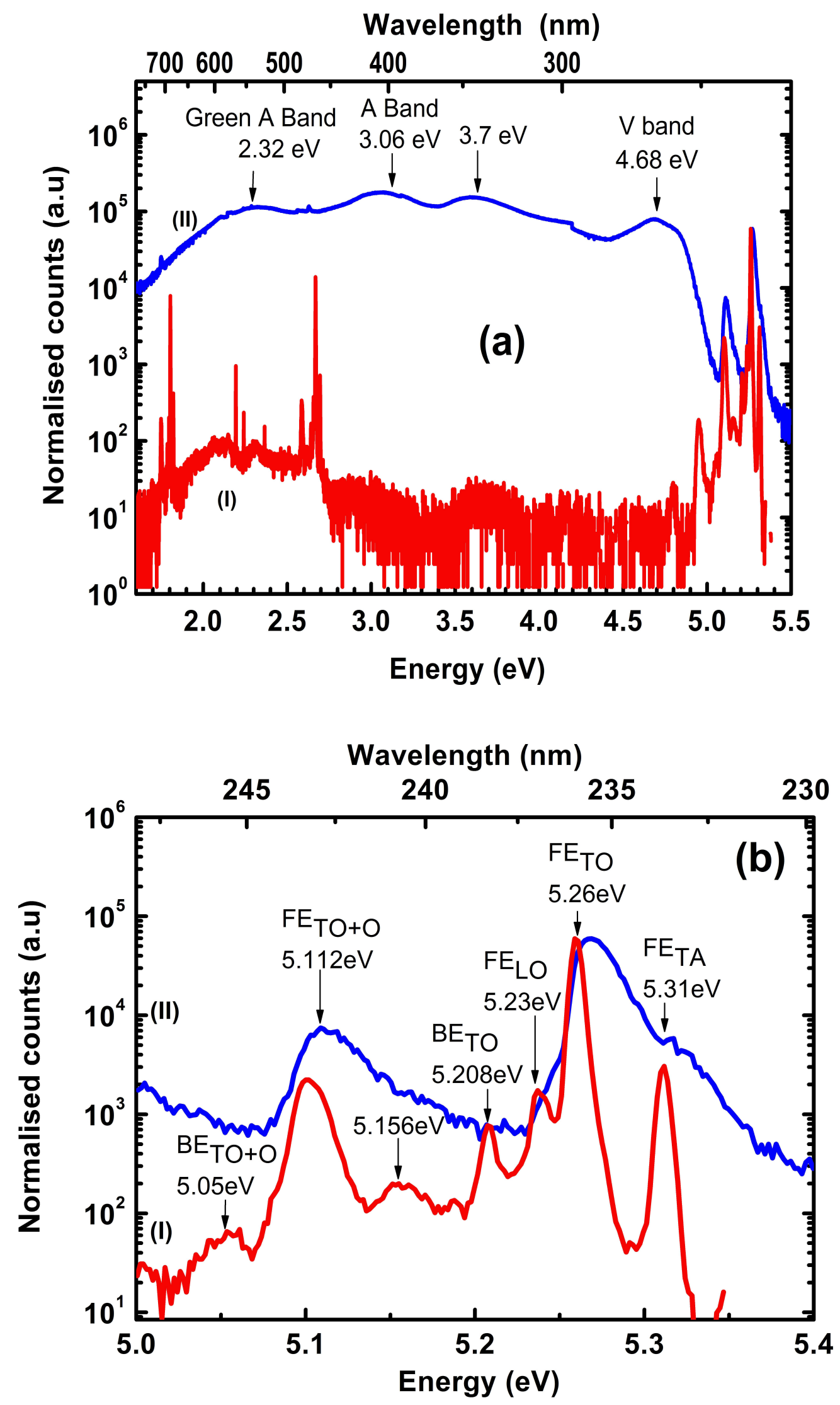
Fig. 2
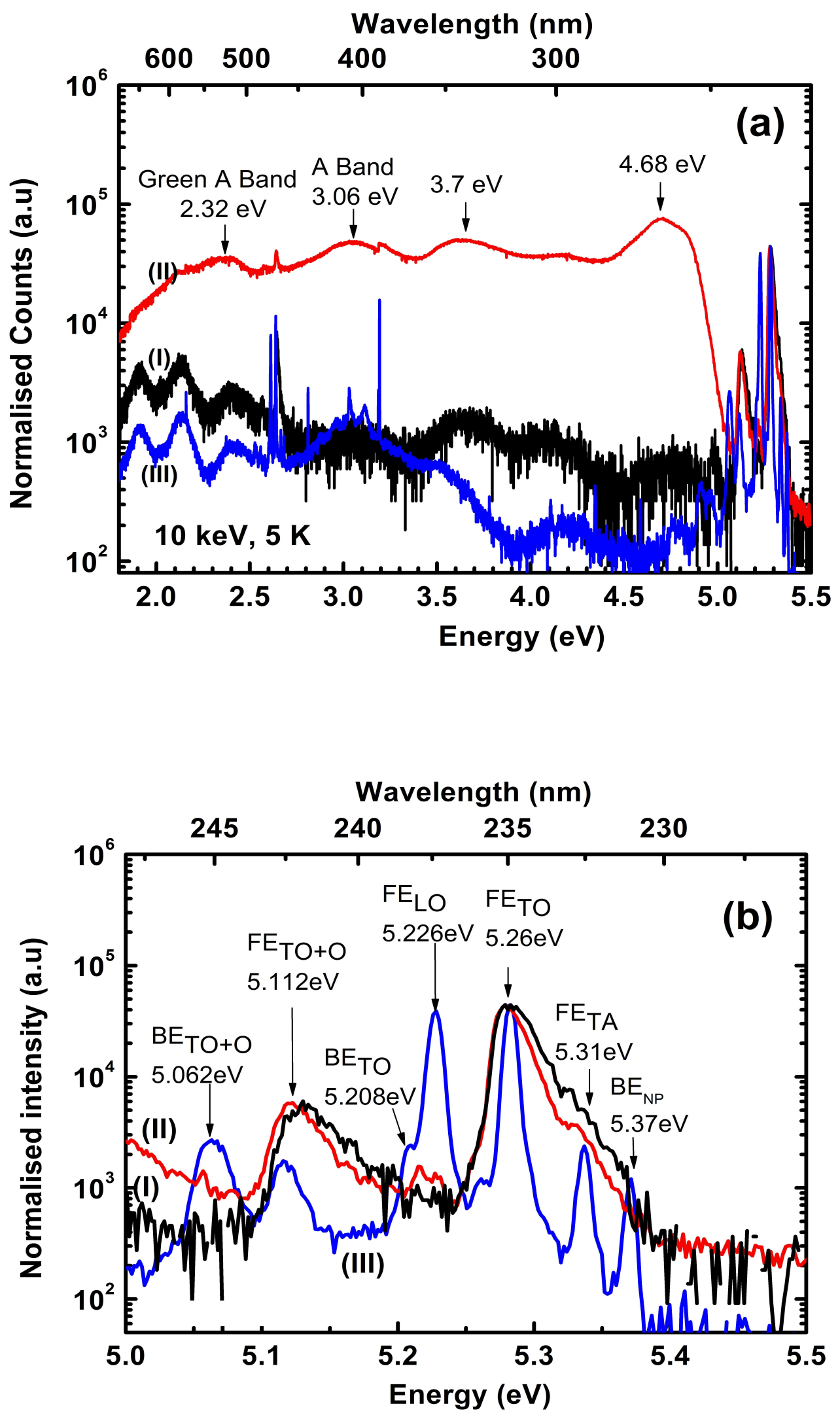
Fig. 3

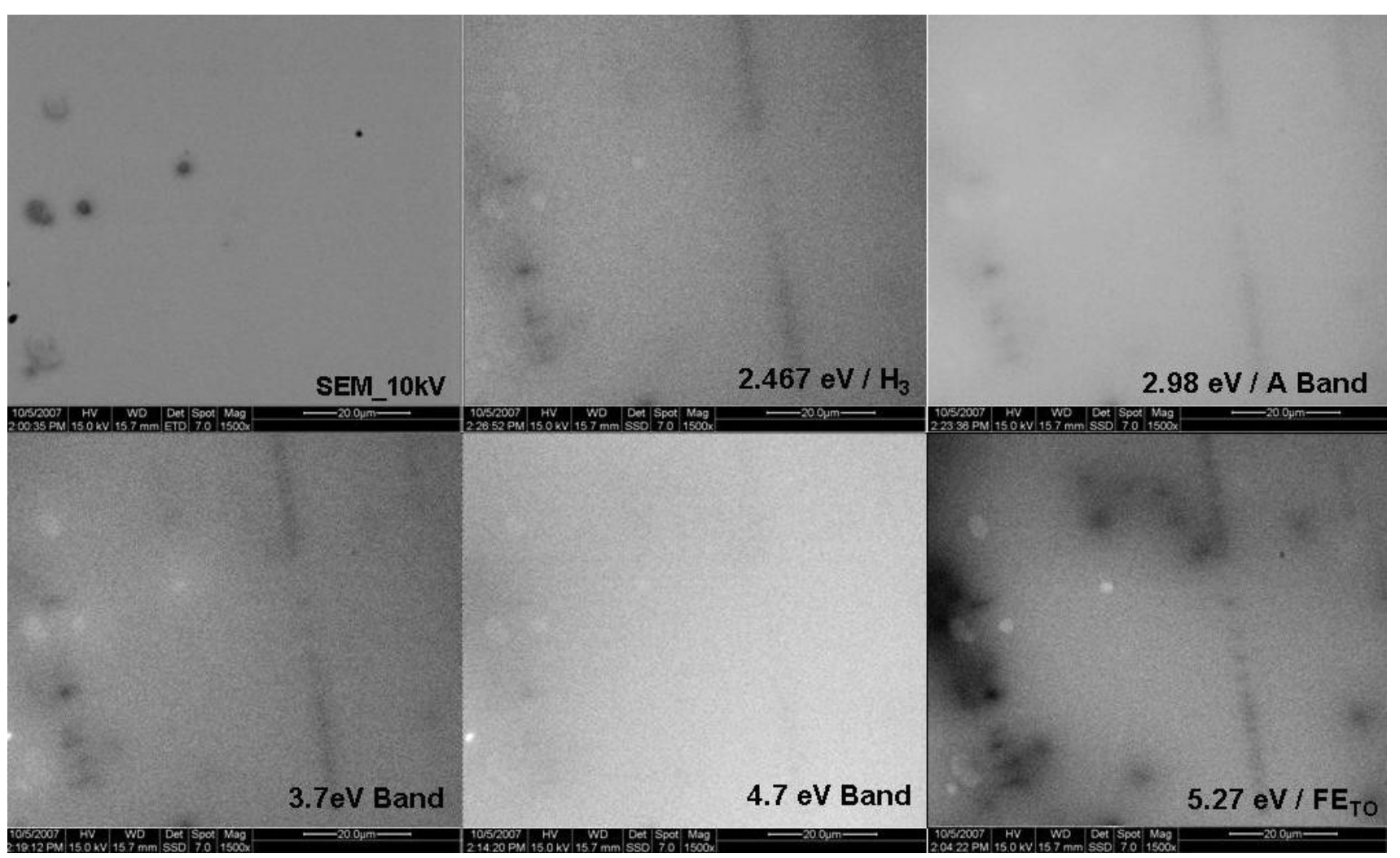

Fig. 4 


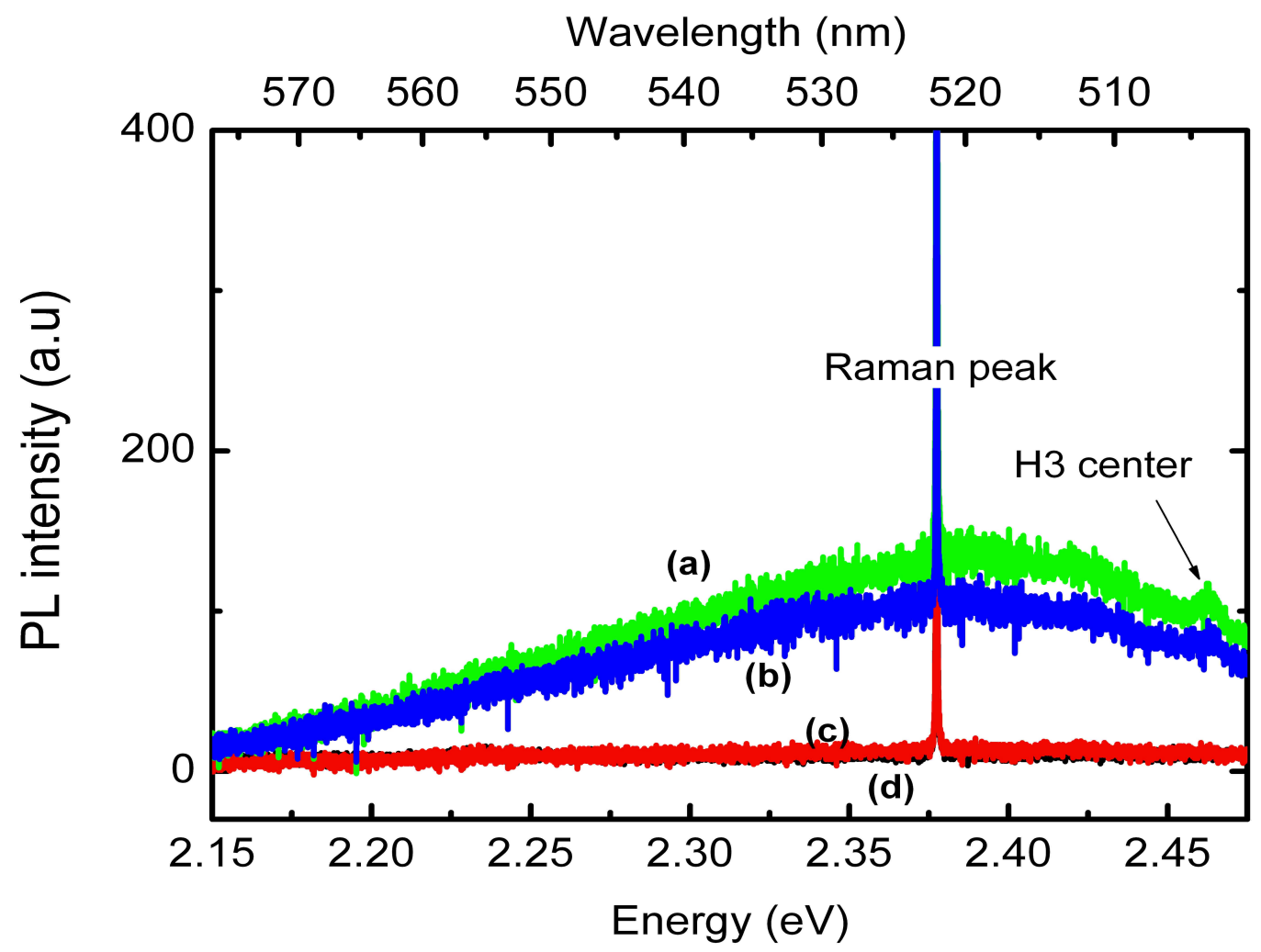

Fig. 5

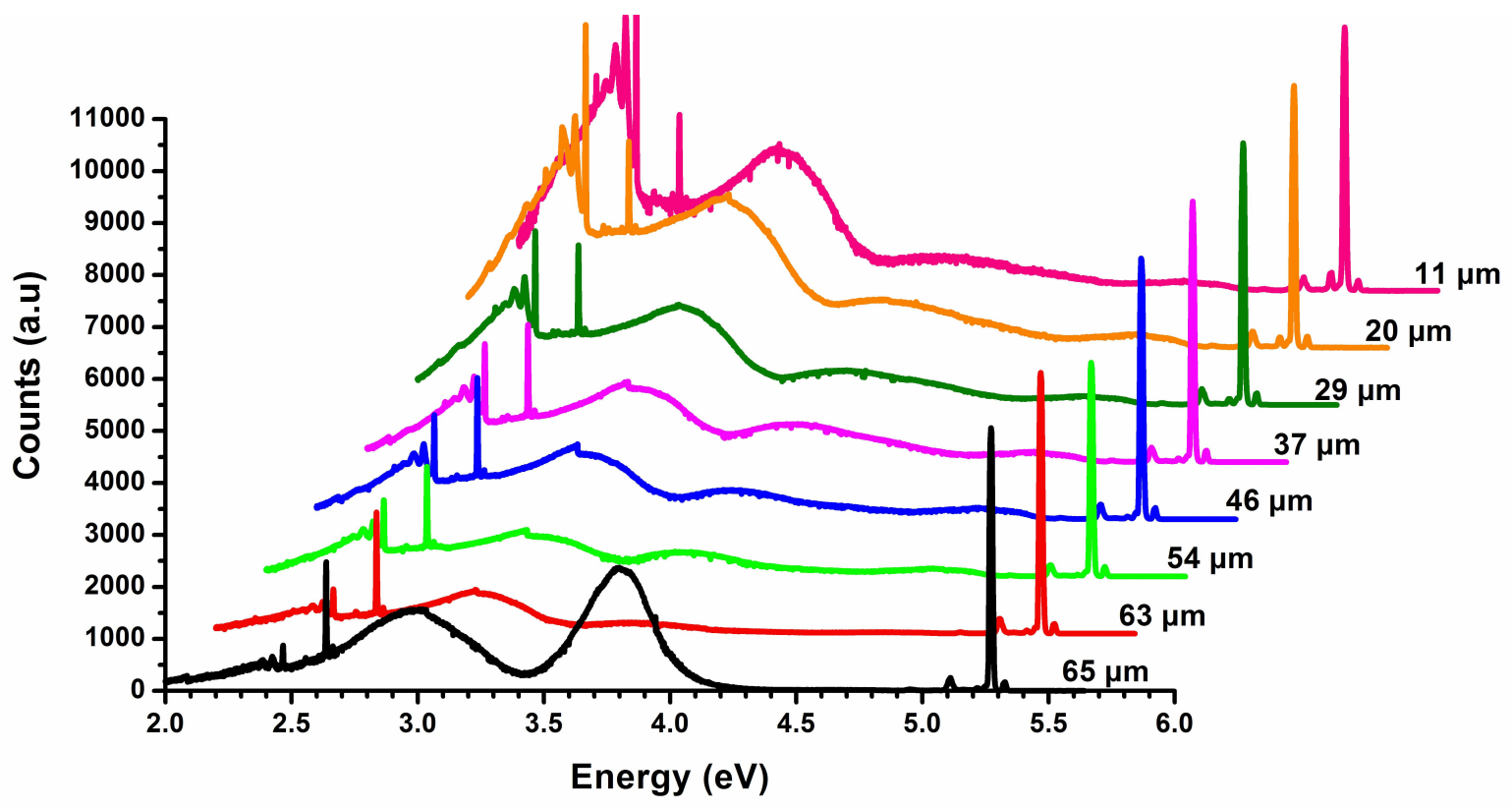


Fig. 6

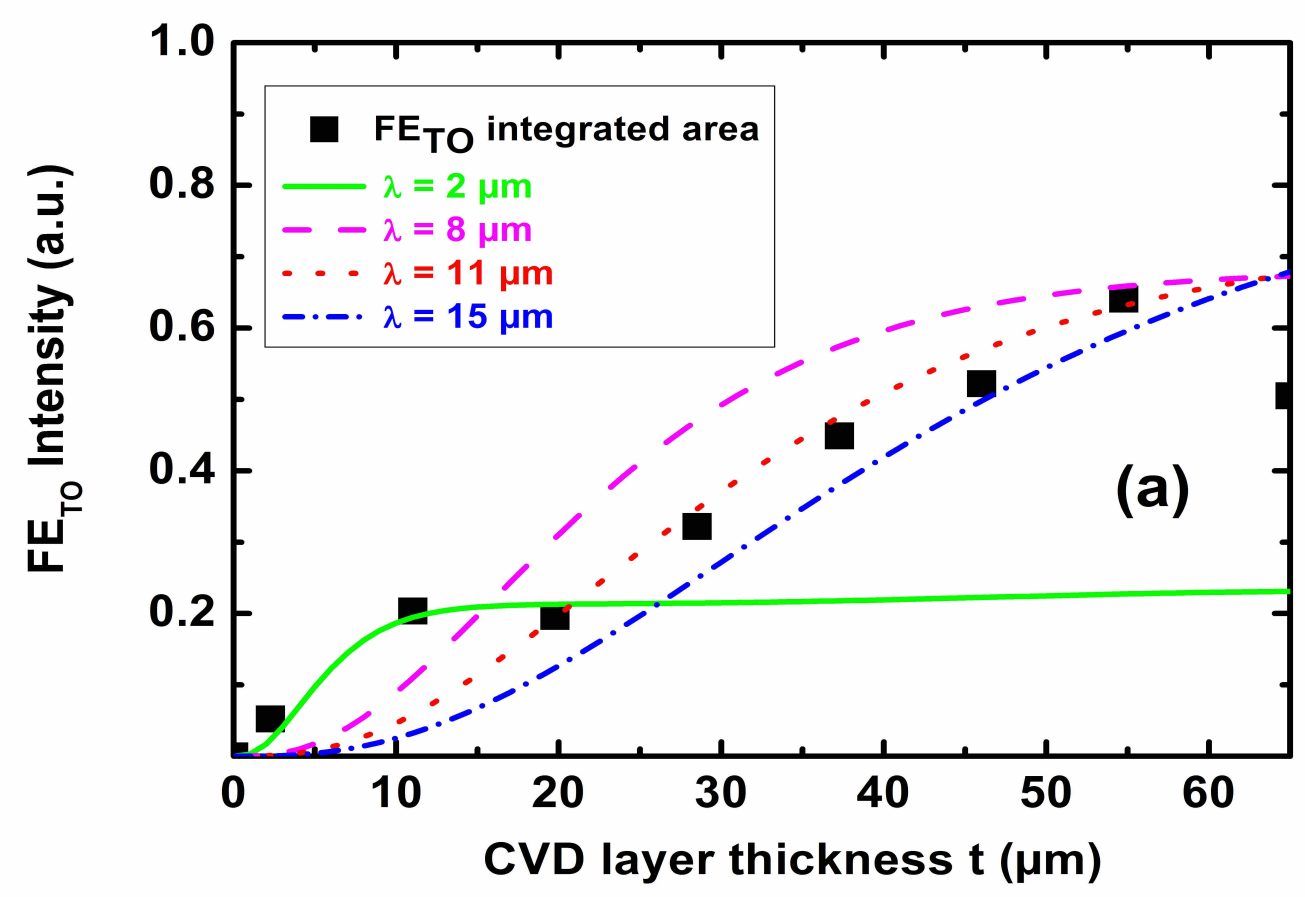

Fig. 7 (a)

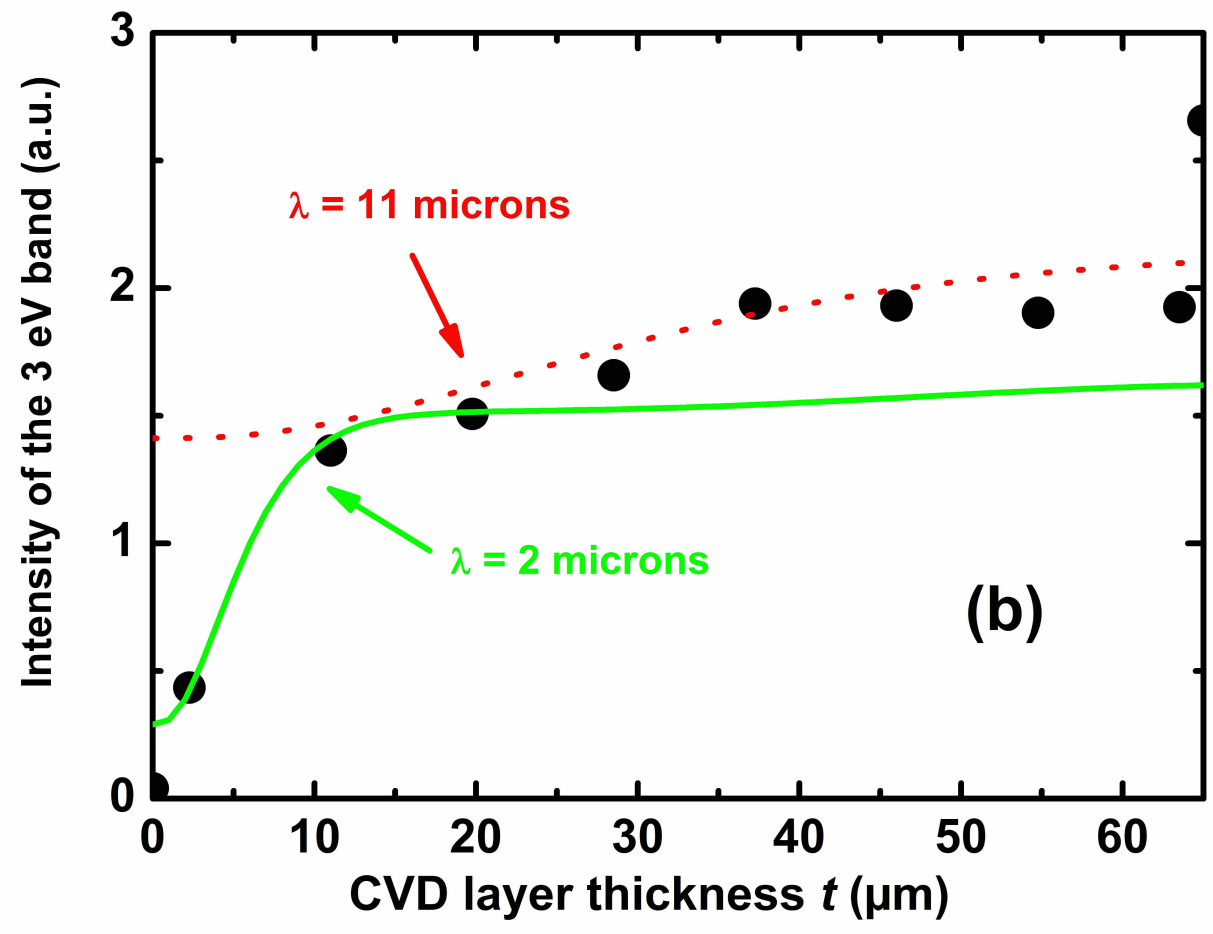


Fig. 7 (b)

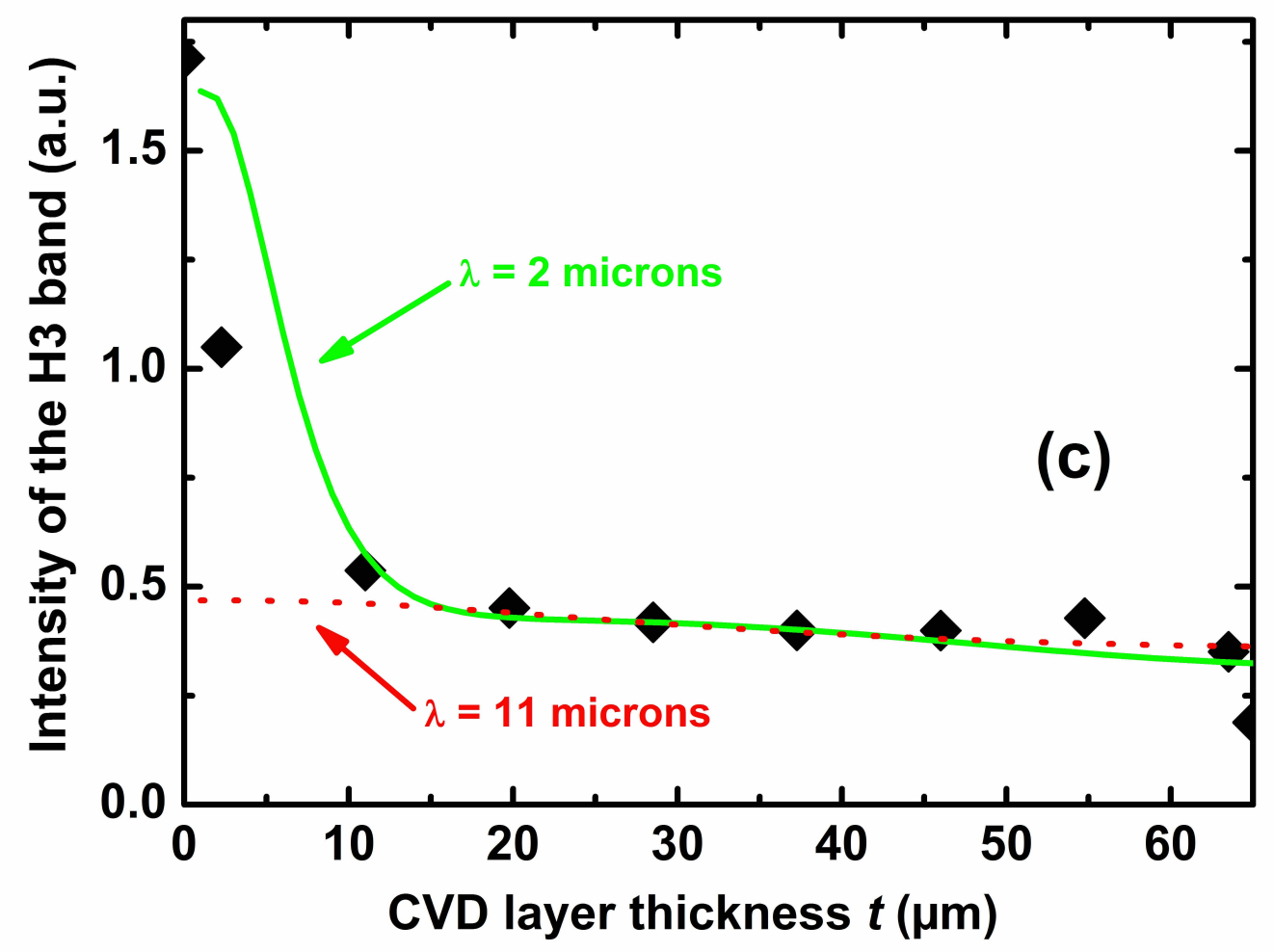

Fig. 7 (c) 